\title{
Avaliação do risco de quedas em pacientes idosos saudáveis
}

\author{
Evaluation of the risk of falls in healthyelderlypatients
}

\author{
Leonardo Contato Balieiro, ${ }^{1,}$ \\ Bruna Soares Vilaça ${ }^{1}$ \\ Ana Paula Tondato Nassif da Trindade ${ }^{1}$ \\ Anderson Santos Carvalho ${ }^{1}$ \\ Luiz Fernando Alves de Castro ${ }^{1}$
}

\section{Resumo}

O objetivo do estudo foi traçar o perfil sócio demográfico dos idosos, avaliar o equilíbrio dos idosos quantificar o risco de quedas pelo índice de Downton e correlacionar o nível de atividades físicas com o risco de quedas. A pesquisa realizada foi do tipo descritivo, transversal, observacional e quantitativa, dentro do PSF Ana Pinto e do FAMA. Foram utilizados o questionário IPAQ e índice de Downton. O índice de Downton apresentou que uma parte da amostra pertencente ao grupo que realizavam atividades físicas obteve risco de quedas. Nota-se neste estudo que a atividade física não influenciou no risco de quedas, ao mesmo tempo nos alertou para a necessidade de continuar estudos sobre esse assunto.

Palavras-chave: idosos, quedas, atividade física.

\begin{abstract}
The aim of the study was to profile demographic partner of the elderly, evaluating the balance of the elderly, to quantify the risk of falls by Downton index and correlate the level of physical activity with the risk of falls. The research was descriptive, cross-sectional, observational and quantitative within the PSF Ana Pinto and FAMA. the IPAQ and Downton index were used. The Downton index showed that part of the sample belonging to the group who performed physical activities obtained risk of falls. It is noted in this study that physical activity did not influence the risk of falls, while alerted us to the need for furtherstudies on thissubject.
\end{abstract}

Key words: elderly, falls, physical activity.
Afiliação dos autores

Centro Universitário do Planalto de Araxá (UNIARAXÁ), Araxá, Minas Gerais, Brasil.

\section{${ }^{*}$ Autor correspondente}

Rua Ana Maria de Rezende, 130 Recanto Bosque, CEP: 38182-808, Araxá, Minas Gerais, Brasil. e-mail: leocontat@gmail.com

Conflito de interesses

Os autores declararam não haver conflito de interesses.

Processo de arbitragem

Recebido: 19/12/2017 Aprovado: 15/04/2018 


\section{Introdução}

A Organização Mundial de Saúde considera idosos, pessoas com idade igual ou superior a 60 anos em países em desenvolvimento e com mais de 65 anos em países desenvolvidos. Aponta ainda que em 2005 o Brasil terá um aumento da população idosa em torno de 32 milhões e ocupará o sexto lugar no mundo ${ }^{1}$.

Para ter uma boa saúde na terceira idade, depende dos hábitos de vida e atividade física ${ }^{2}$.

Esse estudo tem o objetivo de avaliar o risco de quedas em pacientes idosos saudáveis.

\section{Métodos}

O presente estudo foi caracterizado como quantitativo, descritivo, transversal e observacional.

Foi realizado no Programa de Saúde da Família Ana Pinto e na Fundação de Assistência à Mulher Araxaense, situados na cidade de Araxál MG, perante a assinatura do termo de autorização pelo responsável do mesmo, no período de maio a julho de 2015

O estudo foi previamente aprovado pelo Comitê de Ética em Pesquisa do Uniaraxá pelo protocolo no ${ }^{0}$ 00450/11. Os participantes foram informados sobre os procedimentos $e$ assinaram a Carta de Informação ao Sujeito da Pesquisa e o termo de consentimento livre e esclarecido de acordo com a resolução 466/2012 que normatiza a pesquisa com seres humanos.

Foram incluídos neste estudo Indivíduos com idade igual ou superior a 60 anos, ambos os gêneros, praticante ou não de atividade física.

Foram excluídos do estudo, indivíduos com alterações do sistema vestibular, com baixo score no mini-exame de estado mental, lesões graves recentes de membros inferiores e coluna, e disfunções motoras graves.

Para a avaliação do nível de atividade física, foi aplicado o Questionário Internacional de Atividade Física (IPAQ). ${ }^{3}$. O questionário pode ser encontrado na versão curta que é composta por 8 questões e versão longa composta por 27 questões, explorando mais campos ${ }^{4,5}$.
Para avaliar as funções cognitivas foi aplicado o MEEM. Esse instrumento tem o objetivo de avaliar funções cognitivas específicas. $\mathrm{Na}$ aplicação do questionário o ponto de corte foi determinado pelo nível de escolaridade. Idosos analfabetos apresentam déficits cognitivos quando a pontuação total for menor que 20 pontos, e os alfabetizados abaixo de 25 pontos eram considerados com déficits cognitivos ${ }^{6}$.

O Índice de Downtonavalia o risco de quedas de acordo com os sinais físicos e mentais. Escore total são 11 pontos, indivíduos que apresentarem escore igual ou superior a três pontos indicam alto risco de quedas ${ }^{7}$.

Os testes e avaliações foram aplicados por apenas um avaliador, evitando assim falsa interpretação dos resultados.

A coleta se iniciou com a explicação sobre o objetivo do estudo e a forma como preencher os instrumentos de pesquisa, fornecidas pelo aplicador dos questionários e sobre os procedimentos envolvidos no trabalho. Primeiramente os participantes assinaram o Termo de Consentimento Livre e Esclarecido, após foi aplicado o questionário IPAQ, MEEM e índice de Downton.

O período para coleta dos dados ocorreu nos meses de junho a agosto de 2015.

Para interpretação dos dados estatísticos e informações foram apuradas e baseadas através de uma planilha eletrônica e dispostas sob forma de gráficos e ou tabelas, utilizando-se para tal o software Microsoft Office Excel Versão 2010.

\section{Resultados}

Participaram do estudo 20 (vinte) idosos sendo 5 (cinco) homens e 15 (quinze) mulheres, com idade média $73 \pm 8$ anos, divididos em dois grupos: sedentários e praticantes de atividade física.

Em relação se os idosos fazem uso de medicamentos, $75 \%$ usam algum tipo de medicamento e $15 \%$ não fazem ingestão.

Com relação ao nível de atividade física $5 \%$ correspondem a indivíduos muito ativos, $30 \%$ ativos, $20 \%$ insuficientemente ativo $B, 15 \%$ insuficientemente ativo $A$ e $30 \%$ sedentários.

Para análise foi separado os grupos e representado os resultados através da tabela 1 e 2 para melhor comparação.

Tabela 1

Resultados do grupo de idosos sedentário pelo do índice de Downton.

\begin{tabular}{cccccc}
\hline Sedentário & Quedas anteriores & Medicamentos & Déf. Senso & Est. Mental & Deambulação \\
\hline 1 & 1 & 2 & 1 & 0 & 0 \\
2 & 1 & 1 & 1 & 0 & 0 \\
3 & 1 & 2 & 1 & 0 & 0 \\
4 & 1 & 2 & 1 & 0 & 0 \\
5 & 1 & 2 & 2 & 0 & 0 \\
6 & 2 & 1 & 0 & 0 \\
\end{tabular}

Tabela 2

Resultados do grupo de idosos ativos pelo índice de Downton

\begin{tabular}{cccccc}
\hline Ativos & Quedas anteriores & Medicamentos & Déf. Senso & Est. Mental & Deambulação \\
\hline 1 & 1 & 3 & 1 & 0 & 0 \\
2 & 1 & 1 & 1 & 0 & 0 \\
3 & 1 & 0 & 1 & 0 & 0 \\
4 & 1 & 1 & 0 & 0 & 0 \\
5 & 1 & 1 & 0 & 0 & 0 \\
6 & 1 & 2 & 2 & 0 & 0 \\
7 & 1 & 1 & 0 & 0 & 0 \\
8 & 1 & 0 & 1 & 0 & 0 \\
1 & 1 & 1 & 0 & 0 & 0 \\
1 & 1 & 1 & 0 & 0 & 0 \\
12 & 1 & 1 & 0 & 0 & 0 \\
13 & 1 & 1 & 0 & 0 & 0 \\
14 & 1 & 2 & 1 & 0 & 0 \\
2 & 2 & 0 \\
2
\end{tabular}

\section{Discussão}

Nosso estudo confirma os dados da literatura apontando uma maior prevalência de mulheres acima de 65 anos $^{8,9}$. Esse fato pode ser explicado pelo maior índice de mortalidade masculina e aumento da expectativa de vida das mulheres ${ }^{10}$.

Com relação ao uso de medicamentos a literatura nos mostra que a maioria dos idosos fazem uso de pelo menos um tipo sendo em muitos casos comum a prática da automedicação ${ }^{11}$. Assim o uso de quatro ou mais drogas aumentam o risco de quedas, devido a doses inapropriadas e por reações adversas.

Estudos mostram que a percepção que os idosos tem sobre a frequência da prática de atividade física se considerando como ativos. Porémquandoaplicado o IPAQ esse número se inverte $^{12,13}$. Pode-se analisar que o percentual de ativos do G2 é menor, o que pode ser explicado pelo fato de os idosos buscarem os exercícios físicos como rotina, e também por não desempenharem outras tarefas de ordem doméstica. Já no G1, pode-se afirmar que os idosos ativos, por mais que não façam 
exercícios físicos, ocupam seus dias com a rotina do lar, trabalho e atenção à família.

Portanto um estudo realizado 2009, separou os idosos de acordo com o gênero e aplicaram o índice de Downton ${ }^{14}$. O grupomasculinoapresentou uma pontuação que variou em quatro pontos. No grupo feminino os valores variaram em seis e cinco pontos. No mesmo estudo observou-se que as alterações oculares e auditivas foram comuns nos idosos.

Em um estudo de 2015, separou os idosos em dois gruposaplicando a Escala de Downton, G1 apresentava baixo risco de quedas e G2 alto risco de quedas. Durante analise dos resultados o G2 apresentou maior instabilidade postural em comparação ao G1. Para quantificar o equilíbrio postural utilizouse plataforma de força que é considerada fundamental e precisa em análise dos distúrbios do equilíbrio ${ }^{15}$.

Nota-se que o risco de quedas nesse estudo está mais relacionado com déficits cognitivos e ao uso de medicação, e atividade física não reduz o risco de quedas.

\section{Conclusão}

Constatamos que a maior porcentagem de idosos na amostra, apresenta nível cognitivo preservado. $O$ índice de Downton apontou que alguns idosos ativos apresentaram risco de quedas, fato que poder ser relacionado à quedas anteriores, uso de medicação associado a déficits cognitivos.

Nota-se neste estudo que a atividade física não influenciou no risco de quedas, ao mesmo tempo nos alertou para a necessidade de continuar estudos sobre esse assunto.

\section{Referências}

1. Silva MC. O processo de envelhecimento no Brasil: desafios e perspectivas. Textos Envelhecimento 2005; 8(1).

2. Oliveira RF et.al. Efeitos do Tai Chi Chuan na aptidãofísica de mulheres adultas e sedentárias. Rev Bras Cienc Mov 2001; $9(3): 15-22$
3. Matsudo $S$ et al. Questionário internacional de atividade física (IPAQ): estudo de validade e reprodutibilidade no Brasil. Rev Bras Ativ Fis e Saúde 2001; 6(2):5-18.

4. Lima BA. Avaliação do nível de qualidade física de uma pequena população adulta do município do Rio de janeiro por meio do IPAQ (Questionário Internacional de Atividade Física) versão curta. Revista Digital, Buenos Aires 2011; 16(162).

5. Silva GSF. et al. Avaliação do nível de atividade física de estudantes de graduação das áreas saúde/biológica. Rev Bras Med Esporte 2007, 13(1).

6. Brucki SMD. et.al. Sugestões para o uso do Mini-Exame do estado mental no Brasil. Arq Neuropsiquiatr 2003; 61(3):777781.

7. Biazus $M$, Balbinot N, Wibelinger L. Avaliação do Risco de Quedas em Idosos. RBCEH 2010; 7(1): 34-41.

8. Pinho TAM. et. al. Avaliação do risco de quedas em idosos atendidos em Unidade Básica de Saúde. Revista Esc. Enfermagem 2012; 46(2): 320-327.

9. Coutinho ES, Bloch KV, Rodrigues LC. Characteristics and circumstances of falls leading to severe fractures in elderly people in Rio de Janeiro, Brazil. Cad Saúde Pública 2009; 25(2): 455-459.

10. Menezes $\mathrm{RL}$ et.al. Estudo longitudinal dos aspectos multidimensionais da saúde de idosos institucionalizados. Rev. Bras. Geriatr. Gerontol. 2011; 14(3).

11. Goulart LS et.al. Consume de medicamentos por idosos de uma unidade básica de saúde de Rondonópolis/MT. Estud. Interdiscipl. Envelhec. 2014; 19(1):79-94.

12. Silva MF. et.al. Relação entre os níveis de atividade física e qualidade de vida de idosos sedentários e fisicamente ativos. Rev. bras. geriatr. gerontol. 2012; 15(4).

13. Alon JR; Garcés SBB. Envelhecimento ativo: a percepção de escolares e idosos. Revista Digital 2009; 14(132).

14. Machado TR. et.al. Avaliação da presença de risco para queda em idosos. Rev. Eletr. Enf 2009; 11(1): 32-38.

15. Oliveira MR. et.al. Elderly individuals with increased risk of falls show postural balance impairment. Fisioter. Mov. 2015; 28(2). 